\title{
Epigenetic activation of immune genes in cervical cancer
}

Nina Milutin Gašperov, ${ }^{1 *}$ Sanja A Farkas, ${ }^{2}$ Torbjörn K Nilsson, ${ }^{3}$ and Magdalena Grce ${ }^{1}$

\author{
${ }^{1}$ Division of Molecular Medicine, Rudjer Boskovic Institute, Zagreb, Croatia \\ ${ }^{2}$ Department of Laboratory Medicine, Örebro University Hospital, Örebro, Sweden \\ ${ }^{3}$ Department of Medical Biosciences/Clinical Chemistry, Umeå University, Umeå, Sweden
}

*Corresponding author: Nina Milutin Gašperov, Rudjer Boskovic Institute, Division of Molecular Medicine, Laboratory for Molecular Virology and Bacteriology, Bijenicka 54, 10000 Zagreb, Croatia; Phone: +385-1-4561110; Fax: +385-1-4561010; E-mail: nmilutin@irb.hr

\begin{abstract}
Immune system provides us protection from infectious pathogens and tumors formation during lifetime. Cervical cancer (CC), and its cause, human papillomavirus (HPV) are both challenges for the immune system. We present here evidence of epigenetic activation of immune system genes in CC. Illumina Infinium Human Methylation $450 \mathrm{~K}$ BeadChip identified genes, which were all significantly hypomethylated in CC tissue versus normal tissue. The GeneMANIA computer program identified a tight network between those genes. The most strongly correlated genes based on their function are immune effectors' process (AIM2, BST2, BTN3A3, and $I L 12 R B 1)$ and response to virus related genes (AIM2, BST2, and IL12RB1). Thus, activation of those genes through demethylation is probably triggered by HPV oncogenes. In conclusion, the immune system of women who do not develop CC is probably activated earlier through DNA demethylation.
\end{abstract}

\section{Keywords}

Immunity, cancer, HPV, gene, hypomethylation 


\section{Highlights}

- Study group included normal, neoplastic and cancer cervical samples.

- Group of immune system genes were significantly hypometylated in cancer samples.

- Genes are well networked and related to HPV infection response.

- We underscore the importance of epigenetically driven immune response.

\section{Acknowledgments}

We are indebted to Drs Mariastefania Antica and Petar Ozretić for their helpful advices during the preparation of this manuscript. Drs Grce and Milutin Gašperov were supported in part by grant from the Croatian Ministry of Science, Education and Sports (Grant number: 0980982464-2510), while Drs Nilsson and Farkas were supported by the Lions Cancer Foundation, Nyckelfonden, and Örebro läns landsting.

\section{Letter to the Editor}

Protection from infectious pathogens largely results from the coordinated interaction of the cells and molecules of the mammalian immune system. Key cellular components of the adaptive immune system are white blood cells. Both B and T lymphocytes share features of adaptive immunity that includes the ability to recognize pathogen components via clonal expression of a unique cell surface receptor. In the 1950s, Burnet and Thomas proposed the concept of immune surveillance of cancer. This physiological function would have the ability to recognize tumor cells as abnormal cells and to destroy them before they develop into malignant, detectable tumors. Tumor immune escape is presented through tumor growth, invasion, and metastasis. Thus, tumor cells developed different mechanisms to escape immune system such as defect of expression of antigens on the tumor cell surface, the production of immunosuppressive molecules and resistance to apoptosis [1].

The third most common cancer among women worldwide is cervical cancer (CC). Up to ten and more years are necessary for the development of precancerous lesions, the cervical intraepithelial neoplasia (CIN) that precede CC [2]. Human papillomaviruses (HPVs) are well established causative agents of $\mathrm{CC}$, and also other anogenital tumors and head and neck carcinomas [2]. To date, more than 150 HPV types have been identified among which 40 infect 
the anogenital tract including 12 having high carcinogenic potential (also named high-risk, HR) [3]. Although a majority of women are infected with HPV during their sexual life, merely a minority develop CC. Actually, only long lasting HR HPV infection will progress to CIN and further to cancer, but only 1/3 of HPV infected women develop an innate immune response to HPV [3]. Thus, it is still unknown why some women develop CC and others not, and what is the extent of the immune system in cervical cancerogenesis.

Generally HPV develops several mechanisms to evade the host's immune system. The presentation of viral antigens to the host immune system is limited as HPV infection is restricted to epithelial cells [4]. Furthermore, HPV does not kill or destroy infected cells but is shed when those cells undergo programmed apoptosis, and in addition, during infection there is no inflammation. Still, despite all the mechanisms of immune evasion that HPV employs, adaptive immunity does play a role in the clearance of the infection. In specific cell-mediated immune response dendritic cells from the cervical epithelium play an important role in recognizing HPV-infected cells and stimulate T helper 1 (Th1) cells, which elicits the production of cytotoxic $\mathrm{T}$ lymphocytes [5]. In addition, innate immunity activates interferon response, macrophages and natural killer (NK) cells and plays a significant role in activating and directing adaptive immunity for the virus clearance [6].

There is emerging evidence suggesting that epigenetic changes such as histone modifications and DNA methylation play a key role in controlling the distinct transcriptional profiles of memory lymphocytes and thus in shaping their function [7]. In addition, those epigenetic mechanisms are probably involved in antigen presentation, self/nonself discrimination, and the balance between tolerance and autoimmunity [8].

Herein, we provide strong evidence that there is an epigenetic activation of genes involved in the immune system response in CC tissue and, at lesser extent, in CIN3 samples compared to normal tissue. Our study group included 20 normal cytological smears, 19 CIN3 and 6 CC. DNA isolation and HPV genotyping was previously described by Milutin-Gasperov et al. [9]. DNA methylation measured by the genome-wide assay Illumina Infinium Human Methylation $450 \mathrm{~K}$ BeadChip (Illumina, Sweden) was recently described by Farkas et al. [10]. Among other findings, we identified a group of genes statistically significantly hypomethylated in CC samples compared with the normal tissue, which all belong to the immune response regulatory genes. This led us to evaluate the relationship between them. For that we used the GeneMANIA prediction server (http://www.genemania.org) and identified the network of 
significantly hypomethylated genes that are AIM2, BST2, BTN3A3, CASP10, CLEC2B, CST7, DAPP1, EDARADD, FAM26F, GBP4, HAVCR2, HLA-DPB1, IGLL1, IL12RB1, MARCO, PIGB, PRTN3, SIPR4, SIGLEC12, SPTA1, STAT5A, and VPREB1 (Figure 1). The most strongly correlated genes based on their function are immune effectors' process (AIM2, BST2, $B T N 3 A 3$, and $I L 12 R B 1$ ) and response to virus, i.e. antimicrobial defense related genes (AIM2, $B S T 2$, and IL12RB1). The AIM2 gene (Absent In Melanoma 2) is an inflammasome sensor that belongs to the PYHIN family. It is known that several microbial invaders (some DNA viruses and some bacteria) can gain access to the cytosol of phagocytic cells and release foreign DNA to trigger AIM2 and caspase-1 activation. BST2 (Bone Marrow Stromal Cell Antigen) is known to play an inhibitory role in viral release from infected immune cells of the host. Further, BTN3A3 (Butyrophilin, subfamily 3, member A3) belonging to a group of major histocompatibility complex (MHC)-associated genes that encode type I membrane proteins, plays a role in the adaptive immune response. Finally, IL12RB1 (Interleukin 12 Receptor, Beta 1) encodes a type I transmembrane protein that belongs to the hemopoietin receptor superfamily. Its product is an interleukin receptor, which binds interleukin-12 (IL12) and is involved in its transduction. IL12 plays an important role in the activities of NK cells and T lymphocytes, and also has anti-angiogenic activity and the role in protective immunity to intracellular bacteria.

In conclusion, the immune system genes are significantly hypomethylated in CC compared to normal tissue indicating that its subsequent activation is most likely a response to HPV oncogenes E6 and E7 that are highly expressed in advanced cancer [2]. Herein, we indicate that the immune system becomes significantly activated in $\mathrm{CC}$ at the epigenetic/DNA methylation level. The question remains: what would happen if immune response related genes were activated earlier by DNA demethylation of specific gene sites, for instance already in lowgrade cervical lesions? Moreover, our findings give a possible explanation why only a minority of HPV positive women develops CC. In other words, the immune system of women who do not develop CC may be activated earlier through DNA demethylation, and consequently elude cancerization. 


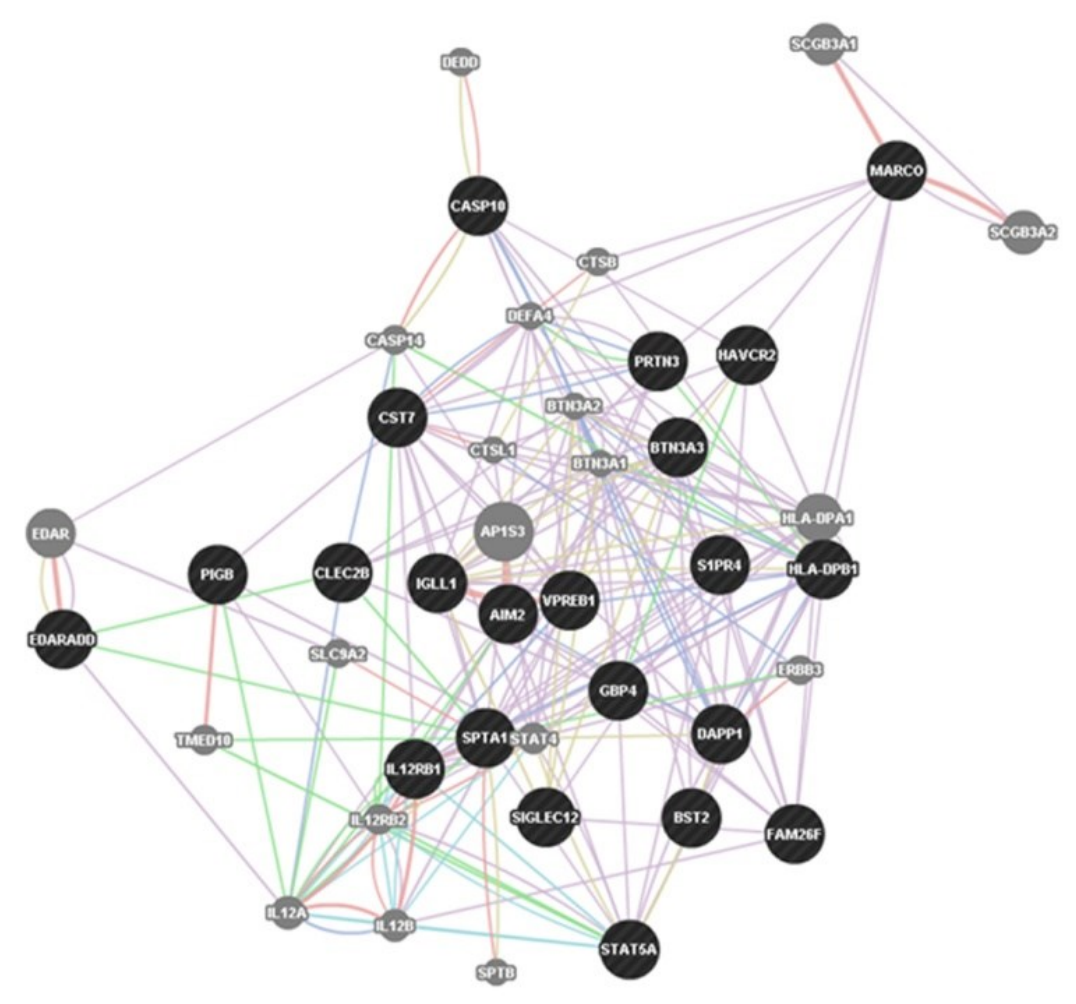

Fig. 1 The tight network of statistically significant hypomethylated genes in cervical cancer tissues compared with the normal tissues according to GeneMANIA prediction server (http://www.genemania.org). The interactions found between these genes are: co-expression (purple lines), physical interactions (pink lines), co-localization (blue lines), genetic interactions (green lines), shared protein domains (grey-yellow lines) and pathway (light blue lines). Genes that are within a black filled circle indicate our depicted hypomethylated genes, while those within a grey filled circle indicate their interactions. 


\section{References}

[1] A. Duray, S. Demoulin, P. Hubert, P. Delvenne, S. Saussez, Immune suppression in head and neck cancers: a review, Clin. Dev. Immunol. 2010 (2010) 701657. doi:10.1155/2010/701657.

[2] IARC, IARC Handbooks of Cancer Prevention: Cervix Cancer Screening, IARC Press, International Agency for Research on Cancer, World Health Organization, Lyon, France, 2005.

[3] J. Doorbar, W. Quint, L. Banks, I.G. Bravo, M. Stoler, T.R. Broker, et al., The Biology and Life-Cycle of Human Papillomaviruses, Vaccine. 30, Supplement 5 (2012) F55-F70. doi:10.1016/j.vaccine.2012.06.083.

[4] M.A. Stanley, Immune responses to human papilloma viruses, Indian J. Med. Res. 130 (2009) 266-276.

[5] S. Man, Human cellular immune responses against human papillomaviruses in cervical neoplasia, Expert Rev. Mol. Med. 1998 (1998) 1-19. doi:10.1017/S1462399498000210.

[6] J. Doorbar, The papillomavirus life cycle, J. Clin. Virol. 32 (2005) 7-15.

[7] N. Weng, Y. Araki, K. Subedi, The molecular basis of the memory T cell response: differential gene expression and its epigenetic regulation, Nat. Rev. Immunol. 12 (2012) 306-315. doi:10.1038/nri3173.

[8] D.R. Fitzpatrick, C.B. Wilson, Methylation and demethylation in the regulation of genes, cells, and responses in the immune system, Clin. Immunol. Orlando Fla. 109 (2003) 37-45.

[9] N. Milutin-Gasperov, I. Sabol, G. Halec, M. Matovina, M. Grce, Retrospective study of the prevalence of high-risk human papillomaviruses among Croatian women, Coll. Antropol. 31 Suppl 2 (2007) 89-96.

[10] S.A. Farkas, N. Milutin-Gašperov, M. Grce, T.K. Nilsson, Genome-wide DNA methylation assay reveals novel candidate biomarker genes in cervical cancer, Epigenetics. 8 (2013). doi:10.4161/epi.26346. 